\title{
Horses for courses: reaching outside drug-like chemical space for inhibitors of challenging drug targets
}

"...attention is turning increasingly to new classes of drug targets that are less amenable to inhibition by conventional drug-like molecules..."

Keywords: ADME • covalent inhibitors $\bullet$ druggability $\bullet$ druglikeness $\bullet$ foldamers

- macrocycles $\bullet$ protein-protein interactions

Periodically, advances in understanding factors that influence success in drug discovery become formulated as rules or guidelines; these become broadly accepted for a time, until new knowledge or a sufficient number of exceptions are identified to highlight the missed opportunities that result from overly rigid adherence. We are coming out of one such cycle now, as it becomes recognized that the concept of 'druglikeness', which has been highly effective in focusing discovery efforts on compounds with potential for achieving good 'pharmaceutical properties' of absorption, distribution, metabolism and excretion (ADME), has at the same time impeded exploration of new pharmacologically useful chemotypes that do not resemble typical known drugs. At the same time, attention is turning increasingly to new classes of drug targets that are less amenable to inhibition by conventional drug-like molecules, such as protein-protein interaction (PPI) interfaces, intrinsically disordered proteins, DNA-binding, carbohydratebinding and some phosphopeptide-binding proteins. This trend has led to growing interest in identifying new chemotypes and drug modalities that possess both an ability to bind to poorly druggable sites and a high potential for good ADME properties.

The features that make a target undruggable by conventional drug-like compounds generally include some combination of the following: a binding site, such as many PPI interface sites, that due to its topology (cavities are too small are too large) cannot generate a large area of intimate contact with a drug-like small ligand; a binding site that is exclusively hydrophobic, and thus lacks the ability to engage in charged or polar interactions with the ligand, as are typically required for high affinity and selectivity; a binding site that has evolved to interact with a highly polar or charged ligand, such as a sugar, a nucleic acid polymer, or a phosphopeptide, and thus is too polar for high complementarity with an amphiphilic drug-like ligand; a site that is intrinsically disordered, such that there is potentially a large entropic cost associated with organizing the binding site around a ligand.

Several promising approaches have been developed to address highly challenging drug targets such as those described above. These approaches exploit compound chemotypes that differ from typical drugs, and thus require us to move beyond conventional views of druglikeness when evaluating prospects for good ADME properties.

\section{Natural product-inspired chemotypes}

The historical success of natural products (NPs) and NP derivatives as drugs provide some of the strongest evidence that chemotypes exist outside of conventional drug-like chemical space that can nevertheless possess good pharmaceutical properties. Indeed, half of the 24 NPs that led to approved drugs during the period 1970-2006 have chemical structure that significantly violate today's definitions of druglikeness [1]. To better exploit the evidently favorable properties of certain NP-like chemotypes for drug discovery, there have been significant efforts aimed at devel-

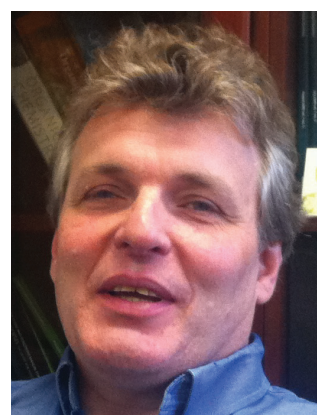

Adrian Whitty

Author for correspondence: Department of Chemistry, Boston University, Boston, MA 02215, USA whitty@bu.edu

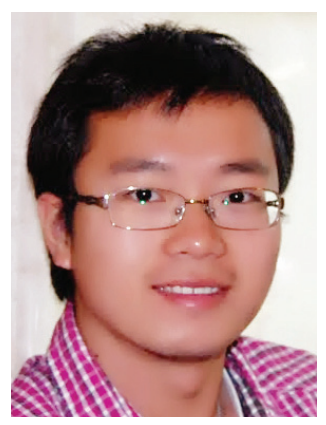

Li Zhou

Department of Chemistry, Boston University, Boston, MA 02215, USA 
oping diverse libraries of such compounds through the application of Diversity Oriented Synthesis methodology [2]. However, advances in how to synthesize NPlike compounds do not address the equally important problem of knowing what to make. Important progress has been made in categorizing the kinds of structures that make up natural products, [3] and in developing libraries that share very general properties of NPs such as an abundance of oxygen versus nitrogen atoms, and a high stereochemical complexity. But such broad guidelines for 'NP-likeness', while undoubtedly useful, nevertheless encompass an enormous volume of chemical space that is far too large to be searched exhaustively. In any case, not all NPs are pharmaceutically useful, and so making compounds that resemble NPs does not in itself guarantee improved prospects for good pharmaceutical properties. Application of the NP-like chemotypes would therefore be greatly facilitated by an improved understanding of what sub-types of NPinspired molecular structures are most likely to interact favorably at different kinds of low druggability binding sites and, separately, what molecular features confer a likelihood of good ADME properties in the context of different NP-like chemotypes.

\section{Macrocycles \& cyclic peptides}

A substantial fraction of larger NP-derived drugs are macrocycles (MCs), suggesting that a macrocyclic structure can help confer good bioavailability on high molecular weight compounds [4,5]. By enabling the use of substantially larger compounds as drugs, MCs provide a promising approach to inhibiting targets, such as many PPI interfaces, for which binding must extend over a large surface area to achieve high affinity [6]. As with NP-derived chemotypes in general, the major barriers to the development of synthetic MCs as tools for drug discovery are the synthetic challenges involved, and also an incomplete understanding of what structural features of MCs are most conducive to the ability to bind to particular target classes as well as to good pharmaceutical properties. Compared to other NP-derived chemotypes, however, there has been rapid recent progress in beginning to understand the pharmaceutical properties and pharmacological potential of macrocycles $[5,8]$. The relatively low flexibility of MCs appears to be a factor, and a proper balance between preorganization and ring flexibility can also give increased binding affinity [5]. An ability to form intramolecular hydrogen bonds, or otherwise shield polar groups, when in a low dielectric environment, also enhances membrane permeability [8]. A tentative set of empirically derived 'design rules' for MCs has recently been proposed, based on analysis of MC drugs and of MC-protein binding modes [6]. But the number of examples available to refine and validate such empirically derived guidelines remains small, emphasizing the need to continue driving toward a more fundamental understanding of the underlying factors.

\section{Peptide foldamers}

Peptide foldamers are molecules intended to mimic elements of protein secondary structure. Some are largely peptidic, while others are nonpeptidic but position key substituents to mimic the placement of amino acid side-chains in the corresponding structural element of a protein. Many PPIs are mediated by recognition of a helix, strand, or loop, and foldamers provide a direct route to designing compounds that compete for binding at such sites. Moreover, foldamers typically are highly selective and, being synthetic, can present a wide range of functionality in the 'side-chain' positions that can increase affinity compared with the natural ligand. For $\alpha$-helix mimetics in particular, the binding epitope typically extends over a relatively large contact area. Consequently, $\alpha$-helix mimetic foldamers tend to be large and 'undruglike'. However, several examples exist of foldamers that are able to enter cells, [9] and indeed a stapled peptide that targets the intracellular protein $\mathrm{p} 53$ is currently in clinical development [10]. In general, however, achieving efficient cell entry, and ADME properties in general, remain key challenges for the foldamer approach.

\section{Covalent inhibitors}

Covalent inhibitors differ from the other chemotypes discussed here in that, generally speaking, they fall within rather than outside conventional descriptions of druglikeness. Their structures differ from those of conventional oral drugs only in possessing a reactive group, usually an electrophile, that can react to form a covalent bond with the target protein. The strong binding conferred by covalent bond formation enables potent inhibition even at poorly druggable sites. Recently, approaches have been developed to generate noncovalent inhibitors that do not depend on interaction with an enzyme's catalytic machinery, and thus can be directed against a much greater range of enzyme and nonenzyme targets [11]. Such compounds, known as 'targeted covalent inhibitors', couple a weak binding moiety that causes the compound to bind transiently but specifically to a particular target site, with a low-reactivity electrophile that covalently reacts with the target only when precisely positioned adjacent to a specific nucleophilic residue - typically a cysteine. Traditional concerns about the potential for toxicity with covalent inhibitors are likely overemphasized, considering the large number of highly successful drugs that work by a covalent mechanism, [11] and these concerns are further alleviated by the 
recognition that the electrophilic 'warhead' can be tuned to possess a low intrinsic reactivity. Because only relatively weak noncovalent binding is required to position the warhead for efficient reaction with the target nucleophilic residue, covalent approaches hold great promise for the inhibition of low-druggability targets. Moreover, covalent inhibitors that react irreversibly intrinsically confer a long target residence time, with its resulting pharmacodynamic benefits [12]. A significant challenge is the need to develop 'warheads' that can efficiently target residues other than cysteine, which would greatly expand the range of target sites to which a covalent approach can be applied.

\section{Future perspective}

A major obstacle to our ability to effectively identify and advance new chemotypes for drug discovery is our poor fundamental understanding of what molecular properties are necessary for pharmacological activity and good ADME properties. Empirically derived property thresholds for druglikeness, based on simple molecular descriptors such as molecular weight, $\operatorname{clog} \mathrm{P}$, number of hydrogen bond donors and acceptors, polar surface area, number of rotatable bonds, number of aromatic rings, etc., can be useful in evaluating compounds that broadly resemble the chemotypes that were used to train the analysis and derive the threshold values, but are of limited use in predicting the pharmaceutical utility of chemotypes that look very different. The fundamental physical principles that govern key properties such as solubility and passive membrane permeability apply similarly to all chemotypes, but the

\section{References}

1 Ganesan A. The impact of natural products upon modern drug discovery. Curr. Opin. Chem. Biol. 12, 306-317 (2008).

2 Schreiber SL. Target-oriented and diversity-oriented organic synthesis in drug discovery. Science 287, 1964-1969 (2000).

3 Koch MA, Schuffenhauer A, Scheck M et al. Charting biologically relevant chemical space: a structural classification of natural products (SCONP). Proc. Natl Acad. Sci. USA 102, 17272-17277 (2005).

4 Driggers EM, Hale SP, Lee J, Terrett NK. The exploration of macrocycles for drug discovery-an underexploited structural class. Nat. Rev. Drug Discov. 7, 608-624 (2008).

5 Giordanetto F, Kihlberg J. Macrocyclic drugs and clinical candidates: what can medicinal chemists learn from their properties? J. Med. Chem. 57, 278-295 (2014).

6 Villar EA, Beglov D, Chennamadhavuni $S$ et al. How proteins bind macrocycles. Nat. Chem. Bio. 10, 723-731 (2014).

7 Bockus AT, McEwen CM, Lokey RS. Form and function in cyclic peptide natural products: a pharmacokinetic perspective. Curr. Top. Med. Chem. 13, 821-836 (2013). formulation of these principles into guidelines based on threshold values of simple molecular descriptors can have very different outcomes for different drug chemotypes. For example, molecular weight appears useful in predicting ADME properties of drug-like compounds. However, it is quite clear that a high molecular weight (i.e., >500 Da) is not in itself a barrier to good pharmaceutical properties $[1,13]$. Therefore, the prominence of molecular weight in metrics for druglikeness is not because size itself is important, but rather because molecular size correlates with other, currently poorly defined, molecular characteristics that do affect ADME properties. Thus, in developing new chemotypes for challenging drug targets we cannot proceed as the proverbial physicist did when predicting the outcome of a horse race; that is, "first, assume all horses are spheres...." Instead, rapid progress will require a deeper understanding of the fundamental principles that govern key pharmaceutical properties, and how these map onto the molecular characteristics of the chemotypes on which we are placing our bets.

\section{Financial \& competing interests disclosure}

A Whitty perfoms ad hoc consulting work, and occasionally receives research funding, from companies interested in developing non-canonical drug chemotypes as drugs. The authors have no other relevant affiliations orfinancial involvement with any organization or entity with a financial interest in or financial conflict with the subject matter or materials discussed in the manuscript apart from those disclosed.

No writing assistance was utilized in the production of this manuscript.

8 Hewitt WM, Leung SS, Pye CR et al. Cell-permeable cyclic peptides from synthetic libraries inspired by natural products. J. Am. Chem. Soc. 137, 715-721 (2015).

9 Bautista AD, Craig CJ, Harker EA, Schepartz A. Sophistication of foldamer form and function in vitro and in vivo. Curr. Opin. Chem. Biol. 11, 685-692 (2007).

10 Aileron Therapeutics Initiates Phase 1 Cancer Study of ALRN-6924 in Advanced Hematologic and Solid Malignancies with Wild Type p53. www.businesswire.com

11 Singh J, Petter RC, Baillie TA, Whitty A. The resurgence of covalent drugs. Nat. Rev. Drug Discov. 10, 307-317 (2011).

12 Copeland RA. The dynamics of drug-target interactions: drug-target residence time and its impact on efficacy and safety. Expert Opin. Drug Discov.

13 Veber DF, Johnson SR, Cheng HY, Smith BR, Ward KW, Kopple KD. Molecular properties that influence the oral bioavailability of drug candidates. J. Med. Chem. 45, 2615-2623 (2002). 